\title{
Emerging role of long non-coding RNA MALAT1 in predicting clinical outcomes of patients with digestive system malignancies: A meta-analysis
}

\author{
CHAO WANG $^{1 *}$, QIAOLING ZHANG ${ }^{2 *}$, YUHUAN HU ${ }^{1}$, JIANSHEN ZHU $^{1}$ and JINYOU YANG ${ }^{1,2}$ \\ ${ }^{1}$ Key Lab of Modern Toxicology of Ministry of Education, School of Public Health, Nanjing Medical University, \\ Nanjing, Jiangsu 211166; ${ }^{2}$ Department of Clinical Medicine and Rehabilitation, Jiangsu College of Nursing, \\ Huai'an, Jiangsu 223005, P.R. China
}

Received March 11, 2018; Accepted November 28, 2018

DOI: $10.3892 / \mathrm{ol} .2018 .9875$

\begin{abstract}
Digestive system malignancies are the most common cancer types worldwide and exhibit an extremely low overall 5-year survival rate. Therefore, clinically applicable biomarkers for predicting clinical outcome are urgently required. Metastasis-associated lung adenocarcinoma transcript 1 (MALAT1) is abnormally expressed in several cancer types. However, to the best of our knowledge, the association between MALAT1 expression and the prognosis of digestive system malignancies remains unknown. Therefore, the current study performed a meta-analysis to comprehensively summarize the association between MALAT1 expression and digestive system malignancies. A total of 1,157 Asian patients from 12 eligible studies [eight studies that investigated overall survival (OS), two studies that investigated disease-free survival and two studies that investigated both indicators] were analyzed. The present results identified a significant association between MALAT1 abundance and poor OS in patients with digestive system malignancies, with a pooled hazard ratio (HR) of 1.62 [95\% confidence interval (CI), 1.35-1.88; $\mathrm{P}<0.001]$. The tumor type, region, sample size and analysis type did not alter the predictive value of MALAT1 as an independent factor for survival. Furthermore, MALAT1 overexpression was an unfavorable prognostic factor for the overall survival of patients with esophageal carcinoma, pancreatic cancer, hepatocellular carcinoma and gastric cancer, with HRs of 1.89 (95\% CI, 1.29-2.49), 1.76 (95\% CI, 0.89-2.63), 1.46 (95\% CI, 0.76-2.17) and 1.41
\end{abstract}

Correspondence to: Professor Jinyou Yang, Department of Clinical Medicine and Rehabilitation, Jiangsu College of Nursing, 9 Keji Road, Huai'an, Jiangsu 223005, P.R. China

E-mail: yangjinyou4518@163.com

*Contributed equally

Key words: metastasis-associated lung adenocarcinoma transcript 1, cancer, digestive system, prognosis, meta-analysis
(95\% CI, 1.04-1.78), respectively. In particular, increased MALAT1 expression levels were significantly associated with decreased OS in patients with colorectal cancer (HR, 3.04; 95\% CI, 1.77-4.31). In conclusion, lncRNA MALAT1 may be a potential prognostic factor for digestive system malignancies in Asian populations.

\section{Introduction}

It is understood that digestive system malignant tumors, which threaten human health in developing and developed countries, are the most common cancer types worldwide (1). The 5-year survival rate is low for digestive system cancer types and the prognosis of patients with cancer of the digestive system remains unfavorable (2). Numerous studies have attempted to generate reliable and persuasive results that may improve the prognosis for patients with digestive system cancer (3-5). However, no specific molecular biomarkers have been routinely applied for these types of tumors. Therefore, novel biomarkers to improve prognosis prediction and novel therapeutic targets are urgently required.

Investigations regarding potential biomarkers of tumorigenesis have typically focused on protein-coding genes (6). With advancements in sequencing technology, it has been estimated that $98 \%$ of the human genome is non-protein coding (7). Long non-coding RNAs (lncRNA) are transcribed RNA molecules with a length $>200$ nucleotides and are located in nuclear or cytosolic fractions with no protein-coding capacity (8). Previous studies have identified that lncRNAs serve important roles in numerous biological processes, including malignant roles in digestive system malignancies by promoting carcinogenesis, metastasis, migration and invasion $(9,10)$. Furthermore, a number of studies have demonstrated that aberrantly expressed lncRNAs are correlated with cell apoptosis, the cell cycle, cell proliferation, cell differentiation, and mRNA transcription and splicing $(11,12)$. Therefore, lncRNAs may be associated with cancer treatment and prognosis, and may serve a role as novel therapeutic targets.

Metastasis-associated lung adenocarcinoma transcript 1 (MALAT1), also termed nuclear-enriched abundant 
transcript 2 or LINC00047, has been the focus of a number of studies due to its association with various cancer types (13). MALAT1 has been revealed to be upregulated in numerous human cancer types, including lung cancer, hepatocellular carcinoma, colorectal cancer (CRC), pancreatic cancer, gastric cancer (GC) and esophageal carcinoma (14-19). Furthermore, a number of epidemiologic studies have reported that MALAT1 is associated with poor survival outcome among patients with advanced lung adenocarcinoma, osteosarcoma, nasopharyngeal carcinoma and breast cancer (20-22). Wang et al (20) identified that the rs3200401 CC genotype of MALAT1 was associated with poor survival for patients with advanced lung adenocarcinoma. Furthermore, Wang et al (22) suggested that MALAT1 serves as an oncogene in osteosarcoma by inhibiting osteosarcoma cell metastasis and proliferation, and inducing cell cycle arrest. A multivariable analysis conducted in the USA confirmed that MALAT1 exhibits independent prognostic significance in a subset of patients with triple-negative breast cancer (21). In addition, previous studies have suggested that the expression of MALAT1 is associated with the development and prognosis of digestive system carcinomas, while in vitro studies have revealed that MALAT1 is involved in the regulation of cancer cell apoptosis and proliferation, and the induction of cell cycle arrest, tumorigenicity and epithelial-mesenchymal transition (EMT) $(14,23,24)$. A previous study demonstrated that MALAT1 mediates certain cellular events underlying metastatic transformation in various cancer cell types (15). Subsequent studies have reported that MALAT1 expression exhibits a role in cell metastasis and the EMT process, which is associated with invasion and metastasis in GC and CRC $(25,26)$. These investigations suggest that MALAT1 should be exploited as a potential prognostic factor for digestive system malignancies.

Previous studies have been limited due to the investigation of single tumor types and small sample sizes. To the best of our knowledge, no meta-analysis has been performed for digestive system malignant tumors. Therefore, the current study performed a meta-analysis to evaluate the association between MALAT1 expression and the prognosis of patients with digestive system malignancies. Furthermore, a comprehensive review concerning the mechanisms of MALAT1 during digestive system malignancies is presented. The present study demonstrated that MALAT1 is associated with the progression of digestive malignant tumors, which suggests it may serve as a prognostic factor for digestive system malignancies.

\section{Materials and methods}

Publication search. To obtain relevant articles for the present meta-analysis, previous studies were searched for in PubMed (https://www.ncbi.nlm.nih.gov/pubmed), Embase (https://www.embase.com/) and Web of Science (http://apps. webofknowledge.com/), and studies published in the Chinese language were identified from the China National Knowledge Infrastructure (http://www.cnki.net/) and WanFang databases (http://www.wanfangdata.com.cn/index.html) up to August 2017. The search strategy involved free-text words and medical subject heading terms. The following key terms were searched: 'long non-coding RNA', 'IncRNA', 'MALAT1', 'Metastasis associated lung adenocarcinoma transcript 1', 'neoplasm', 'cancer', 'tumor', 'colorectal cancer', 'esophageal carcinoma', 'pancreatic cancer' 'hepatocellular carcinoma', 'gastric cancer', 'prognosis', 'survival', 'mortality', 'follow-up', 'outcome' and 'predictor'. The bibliographies of the retrieved articles and relevant reviews, as well as conference reports, were also checked for other eligible studies.

Inclusion and exclusion criteria. Eligible studies had to meet the following criteria: i) Diagnosis with digestive system cancer must be histopathologically confirmed; ii) the expression level of MALAT1 was examined in malignant tissues; iii) the association between the expression level of MALAT1 and patient survival time was comparatively analyzed; iv) a description of MALAT1 expression level measurement, including quantitative polymerase chain reaction, in human tissues; v) a description of the cut-off value of MALAT1; vi) the hazard ratios (HRs) and $95 \%$ confidence intervals (CIs) for survival rate was reported or could be calculated from the published data; and vii) the more comprehensive or recent study was selected when there were duplicate studies. The exclusion criteria were as follows: i) studies without usable data; ii) animal studies, case reports, letters, reviews and conference abstracts; iii) meta-analysis studies; iv) studies that included patients with benign tumors in the digestive system; and v) studies that did not contain HRs, 95\% CIs, P-values or raw data.

Data extraction and quality assessment. The extraction of relevant data from the eligible studies was independently performed by two individuals. The extracted data included the first author, year of publication, country, cancer type, sample size, Tumor-Node-Metastasis stage (27), follow-up months, adjuvant therapy prior to surgery (yes or no), cut-off value, method of MALAT1 expression level measurement, survival rates [overall survival (OS) and disease-free survival (DFS)], HRs and 95\% CIs. If they were not present, HR estimates were reconstructed by extracting several survival rates at specific times from Kaplan-Meier curves, as described previously $(28,29)$, using Engauge Digitizer software version 4.1 (http://digitizer.sourceforge.net/). The quality of eligible studies was assessed using the Newcastle-Ottawa quality assessment scale (NOS) (http://www.ohri. ca/programs/clinical_epidemiology/oxford.asp), as presented in Table I. NOS criteria scores range from 0 (lowest) to 9 (highest). Typically, an NOS score $\geq 6$ is considered to indicate a high-quality study. Disagreements were discussed with other independent investigators.

Statistical analysis. HRs and 95\% CIs were extracted to indicate the association of MALAT1 expression with the survival of patients with digestive system malignancies. A HR value not equal to 1 was considered statistically significant, while a HR $>1$ indicated a worse survival outcome for the group with an increased MALAT1 expression level. Statistical heterogeneity among these studies was tested using Q-test, and the $\mathrm{I}^{2}$ statistic was performed to quantify the heterogeneity across studies. An $\mathrm{I}^{2}$ statistic $>50 \%$ and a $\mathrm{Q}$-test result with $\mathrm{P}<0.05$ indicated strong heterogeneity 
ONCOLOGY LETTERS 17: 2159-2170, 2019

2161

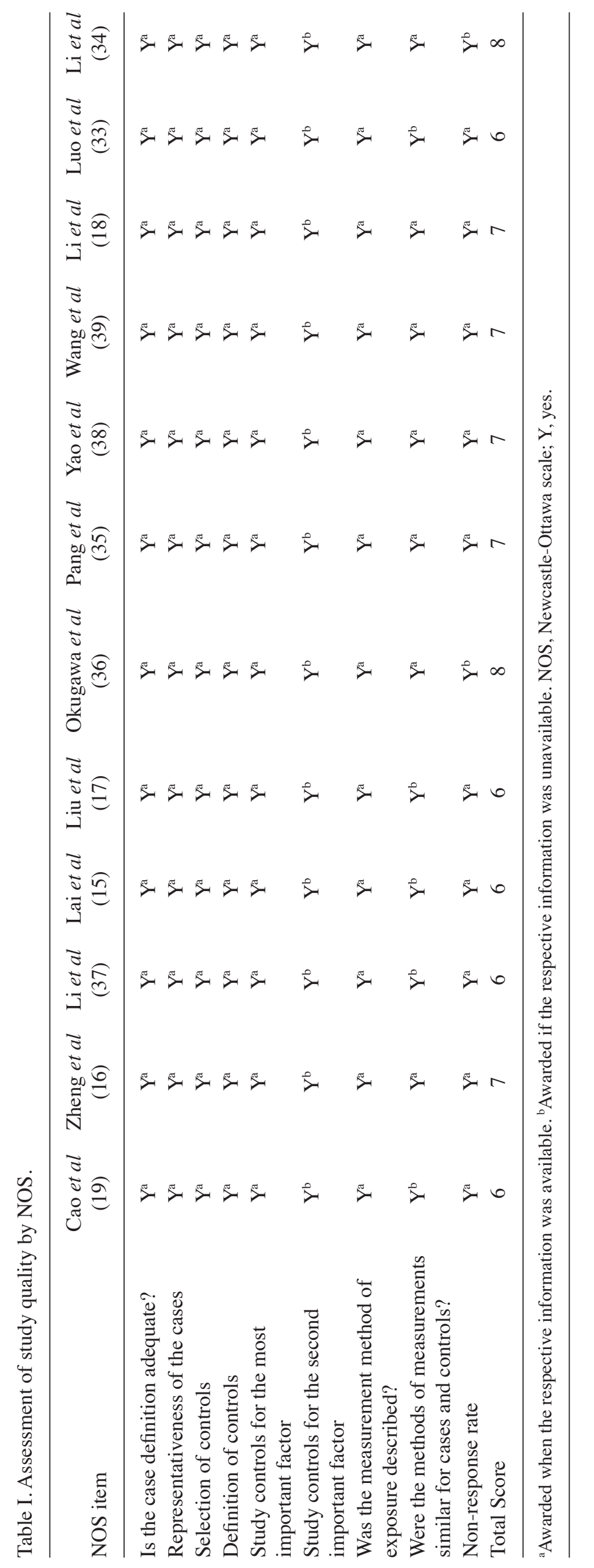


across studies (30). The random-effects model was used to estimate the pooled HR, and if it was more appropriate, a fixed-effect model was used. Furthermore, the influence of heterogeneity was minimized by classifying studies into subgroups according to the following characteristics: Tumor type, histology type, country, sample size and analysis type. Furthermore, a sensitivity analysis was performed, and Galbraith plots were generated to identify the source of heterogeneity. Potential publication bias was tested using Begg's and Egger's tests (31). These were used to detect publication bias when the publications were tendentiously deleted (32). All meta-analyses were performed using Stata 12.0 software (StataCorp LP, College Station, TX, USA). $\mathrm{P}<0.05$ was considered to indicate a statistically significant result.

\section{Results}

Literature search and study characteristics. According to the criteria, a total of 138 references were retrieved in the initial search following meticulously screening. Finally, 12 publications were categorized as eligible for the present meta-analysis to investigate the prognostic value of MALAT1 in digestive system cancer types. The detailed selection steps are summarized in the flow chart presented in Fig. 1. Among the 12 studies, three studies investigated hepatocellular carcinoma $(15,33,34)$, two studies investigated pancreatic cancer $(17,35)$, two studies investigated GC $(18,36)$, two studies investigated CRC $(16,37)$ and three studies investigated esophageal carcinoma $(19,38,39)$. A total of 1,157 patients with survival data were included in the present meta-analysis, with a mean sample size of 96.42 (range, 32-150). The 12 studies all took place in Asia, 11 studies were performed in China and one study was performed in Japan, and study design details were available from all. The overall time period of all 12 studies was from 2012 to 2017. The main characteristics of the studies are summarized in Table II. The participants in all studies were categorized into two groups, a high MALAT1 expression group and a low MALAT1 expression group. The method used to determine the MALAT1 cut-off value varied, as presented in Table II. A total of ten studies reported the OS rate and four studies reported the DFS rate. HR and 95\% CI values were extracted directly from seven studies, while HRs for five studies were extrapolated by graphical representations of the survival curve. The quality assessment of eligible studies was scored using the Newcastle-Ottawa quality assessment scale (Table I).

The prognostic value of MALAT1 in digestive system malignancies. A total of ten studies reported the OS of 1,052 patients, according to MALAT1 expression levels. Heterogeneity analysis revealed no heterogeneity among these studies, therefore, the fixed-effects model $\left(\mathrm{I}^{2}, 0.0 \% ; \mathrm{P}=0.528\right)$ was assessed in the meta-analysis. Overall, the pooled results confirmed a significant association between high MALAT1 expression and poor OS in digestive system malignancies, with a HR value of 1.62 (95\% CI, 1.35-1.88; P<0.001; Fig. 2). Although there was no significant heterogeneity among the studies, a subgroup meta-analysis was conducted on tumor
138 studies identified by the following database: Pubmed (102), Embase (12), Web of science (22), CNKI (1), Wanfang database (1)

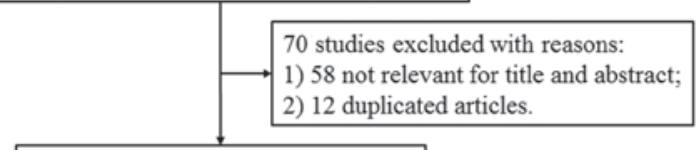

68 articles screened for further abstract review

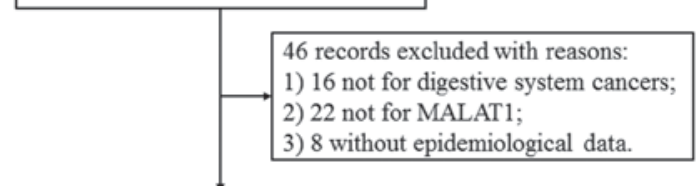

22 full-text articles assessed for eligibility

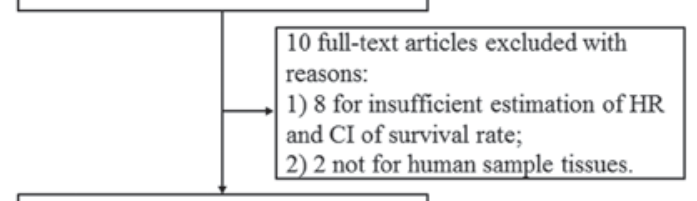

12 articles were included in qualitative synthesis (meta-analysis)

Figure 1. Search strategy and study selection. HR, hazard ratio; CI, confidence intervals; CNKI, China National Knowledge Infrastructure.

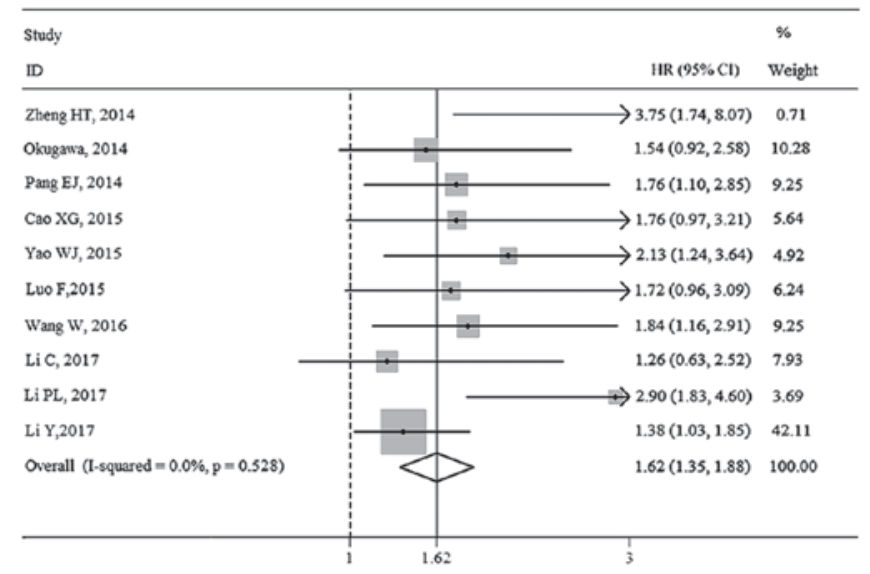

Figure 2. Forest plot of studies that evaluated the pooled HRs of the expression level of MALAT1 and overall survival in digestive system malignancies. The point estimate was bounded by a $95 \%$ CI. Each square represents the HR point estimate and its size is proportional to the weight of the study. The solid line represents the overall survival summary estimate, in which $95 \% \mathrm{CI}$ is given through its width. The dotted vertical line demonstrates the null value (hazard ratio=1), which represents no increased risk for the outcome. HR, hazard ratio; CI, confidence interval.

type, country, sample size and analysis type (Table III). A significant association was identified between the expression of MALAT1 and the OS of patients with digestive system malignant tumors in China (HR, 1.63; 95\% CI, 1.34-1.91). The association between MALAT1 and OS was significant in studies with a sample size $\geq 100$ ( HR, 1.57; 95\% CI, 1.27-1.88; $\mathrm{P}<0.001)$ and $<100$ (HR, 1.76; 95\% CI, 1.21-2.31; $\mathrm{P}<0.001)$. Furthermore, MALAT1 was also identified to be significantly associated with OS of patients in studies with multivariate analysis (HR, 1.51; 95\% CI, 1.19-1.84; $\mathrm{P}<0.001)$ 


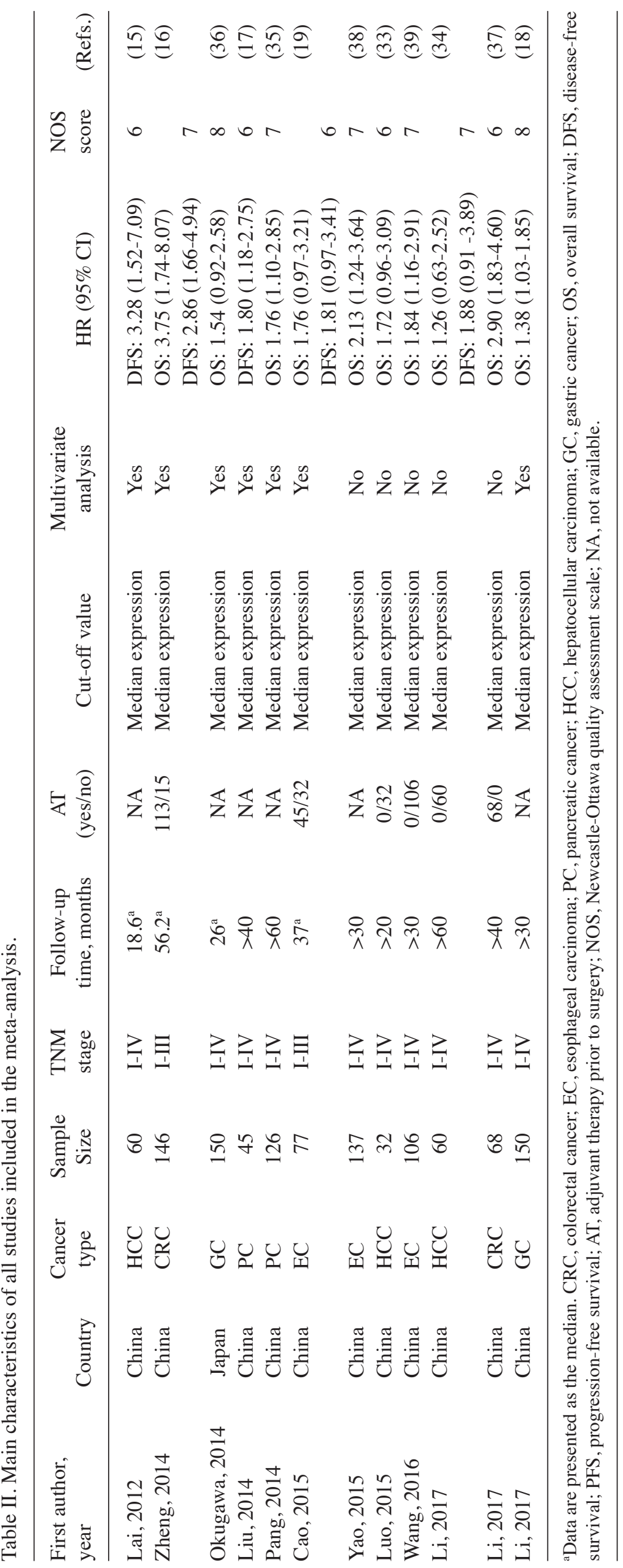




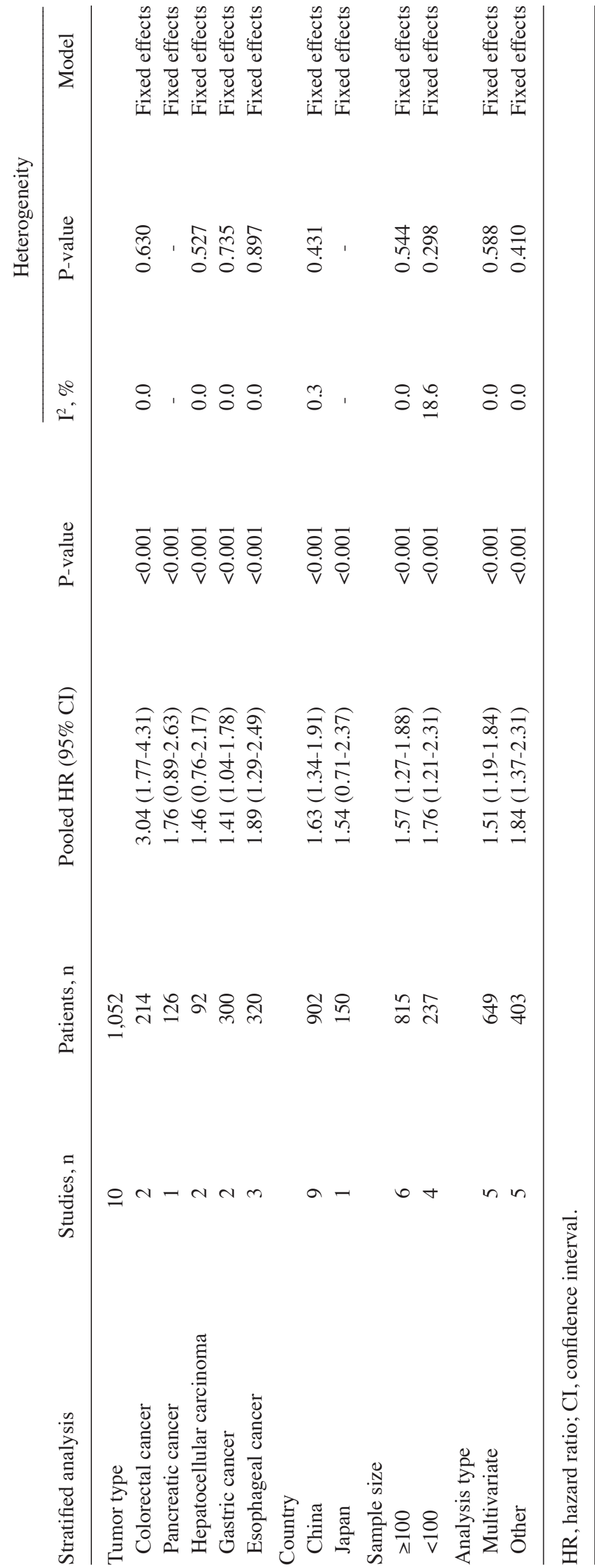




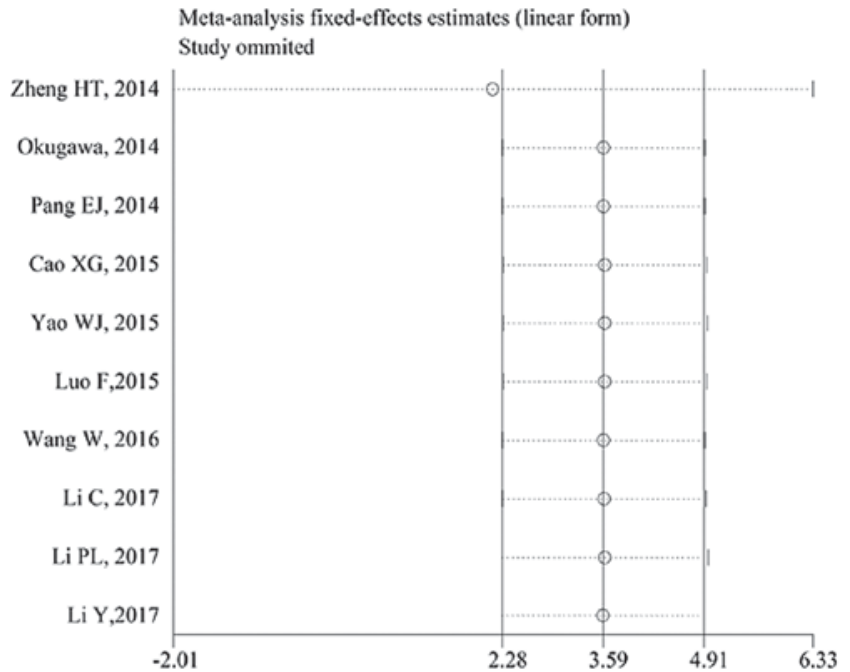

Figure 3. Sensitivity analysis of the meta-analysis. The solid line represents the meta-analysis fixed-effects estimates, in which the $95 \%$ confidence interval is given through the width of the dotted horizontal line. The circle represents each study.
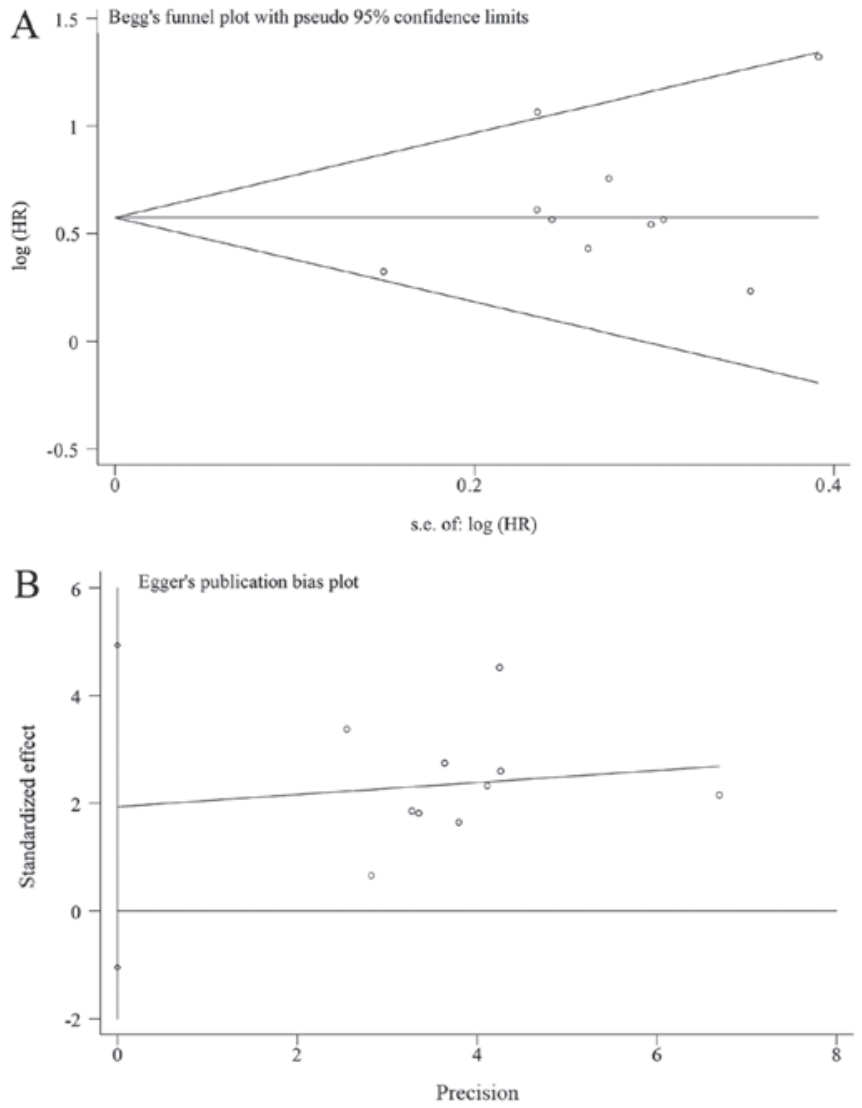

Figure 4. Funnel plot for identifying publication bias. (A) Begg's funnel plot analysis for publication bias. (B) Egger's funnel plot analysis for publication bias. Each point represents a separate study. HR, hazard ratio; s.e., standard error.

and non-multivariate analysis (HR, 1.84; 95\% CI, 1.37-2.31; $\mathrm{P}<0.001)$. Furthermore, sensitivity analysis indicated the robustness of the meta-analysis results (Fig. 3).

Publication bias was assessed using Begg's funnel plot (Fig. 4A) and Egger's test (Fig. 4B). The shapes of the funnel plot were symmetrical. Therefore, publication bias was not identified by Begg's test $(\mathrm{z}=0.18, \mathrm{P}=0.858)$ or Egger's test $(\mathrm{t}=1.49, \mathrm{P}=0.174)$. In studies with DFS data, a high expression level of MALAT1 was observed to be associated with poor DFS (HR, 2.01; 95\% CI, 1.41-2.61) and no significant heterogeneity was identified $\left(\mathrm{I}^{2}=0 \% ; \mathrm{P}=0.53\right)$ (Fig. 5A). Furthermore, subgroup analysis for DFS was not performed due to a limited number of studies but sensitivity analysis was performed (Fig. 5B) and publication bias was assessed (Fig. 5C-D).

Independent prognostic value of MALAT1 for OS in different cancer types. The analysis of subgroups suggested that no significant heterogeneity existed among the studies for the five main types of digestive system cancers $\left(\mathrm{I}^{2}=0.0 \%\right.$; $\mathrm{P}>0.05$; Fig. 6). Therefore, the fixed-effects model was utilized to combine HRs with the corresponding 95\% CI. Notably, it was identified that MALAT1 was a significant prognostic indicator for the OS of patients with CRC (HR, 3.04; 95\% CI, 1.77-4.31; $\mathrm{P}<0.001$ ), pancreatic cancer (HR, 1.76; 95\% CI, 0.89-2.63; $\mathrm{P}<0.001)$, hepatocellular carcinoma (HR, 1.46; 95\% CI, 0.76-2.63; P<0.001), GC (HR, 1.41; 95\% CI, 1.04-1.78; $\mathrm{P}<0.001)$ and esophageal cancer (HR, 1.89; 95\% CI, 1.29-2.49; $\mathrm{P}<0.001)$. Furthermore, the subgroup analysis revealed that the aforementioned factors were not associated with the predictive value of MALAT1. Furthermore, the pooled meta-analysis results confirmed that high MALAT1 expression level in digestive cancer tissue was a significant independent predictor of poor $\mathrm{OS}$ in numerous types of digestive system malignancies.

Systematic review of the molecular mechanism. The mechanism of action for MALAT1 on the development of digestive system malignancies has been investigated in numerous in vivo and in vitro studies. The most recent studies have demonstrated that the expression of MALAT1 is associated with the development and prognosis of digestive system cancer, while in vivo and in vitro studies have indicated that MALAT1 is involved in the regulation of cancer cell apoptosis and proliferation, and the induction of cell cycle arrest, tumorigenicity, migration, invasion and metastasis (Fig. 7). MALAT1 acts as a molecular sponge of miR-146b-5p and downregulates its expression in hepatocellular carcinoma. miR-146b-5p targets TRAF6-mediated Akt phosphorylation and may suppress the expression of Bcl-2 and Bcl-xL, therefore decreased expression of miR-146b-5p is associated with increased cancer cell proliferation and lower rates of cell apoptosis (34). In addition, MALAT1 significantly induces the proliferation and metastasis of cancer cells in vitro and in vivo through the activation of the Wnt, extracellular-signal-related kinase/mitogen activated protein kinase, Erb-B2 receptor tyrosine kinase 3 and c-Myc signaling pathways (18). A number of studies have indicated that p53 is the main downstream mediator for MALAT1 activity and MALAT1 may be regulated by SP1 nuclear transcription factor/latent transforming growth factor $\beta$-binding protein 3 and transforming growth factor- $\beta$, which disrupts the replication of the S-phase, as well as the cell population of G1 and G2/M cells (40). Furthermore, MALAT1 promotes tumorigenicity in GC cells by recruiting splicing factor 2 (SF2)/alternative splicing factor (ASF), 
A

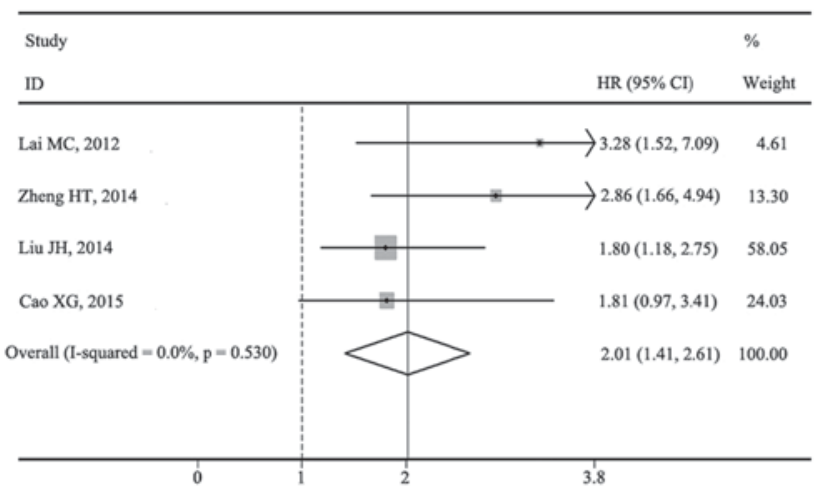

$\mathrm{B}$

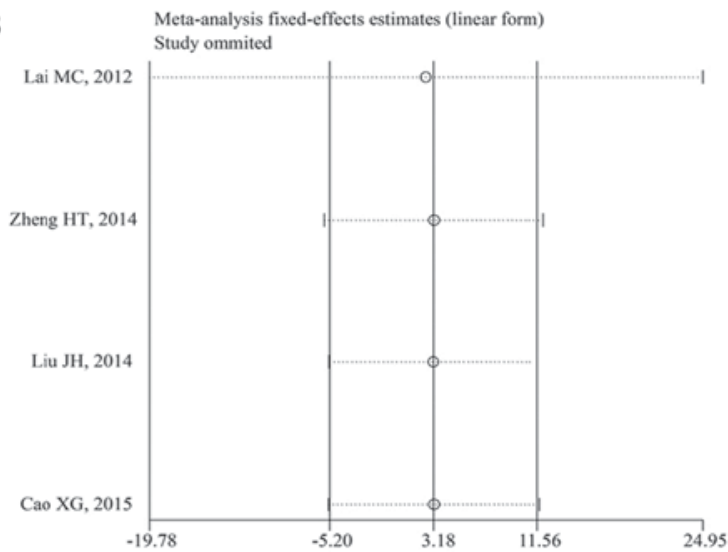

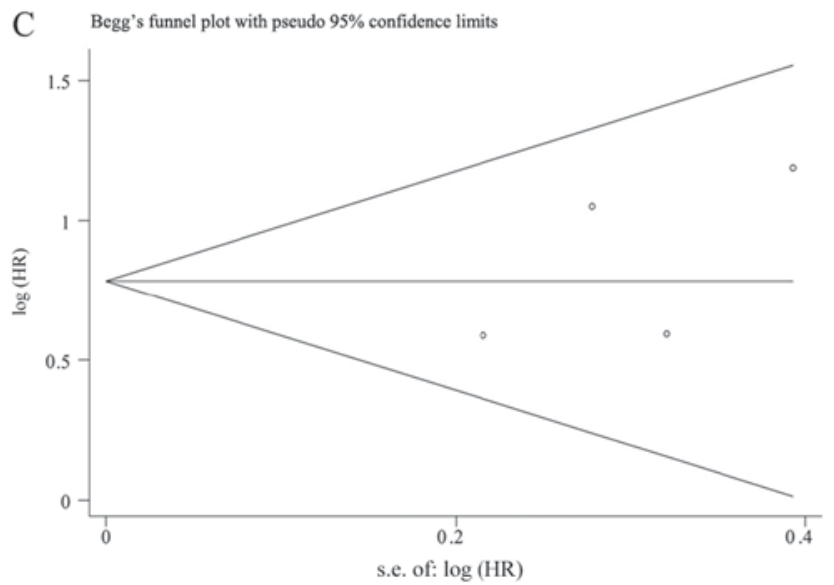

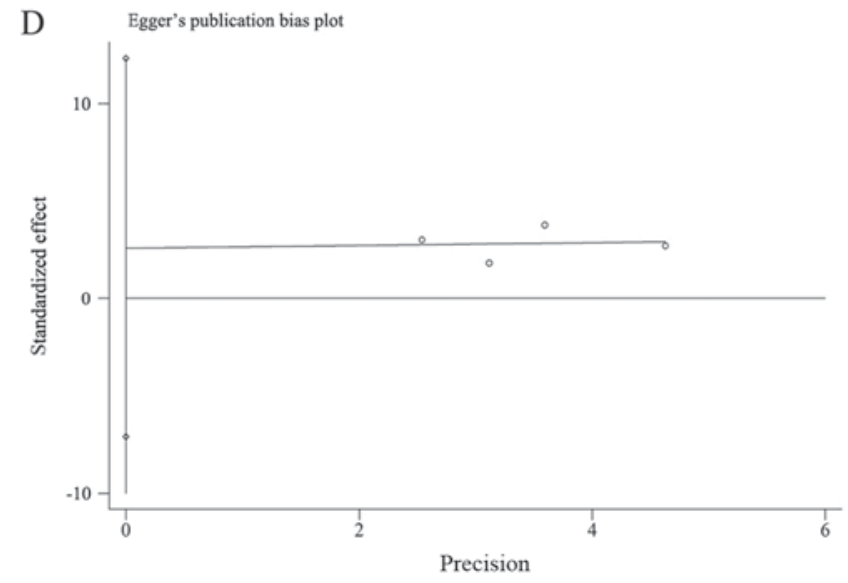

Figure 5. Meta-analysis of pooled HRs to analyze the association between high expression level of MALAT1 and DFS. (A) Forest plot of the studies with DFS data. The solid line represents the overall summary estimate, in which the 95\% CI is given through its width. The dotted vertical line demonstrates the null value (hazard ratio=1), which represents no increased risk for the outcome. (B) Sensitivity analysis. The solid line represents the meta-analysis fixed-effects estimates, in which the 95\% confidence interval is given through the width of the dotted horizontal line. The circle represents each study. (C) Begg's funnel plot analysis. (D) Egger's funnel plot analysis. HR, hazard ratio; CI, confidence interval; DFS, disease-free survival; s.e., standard error.

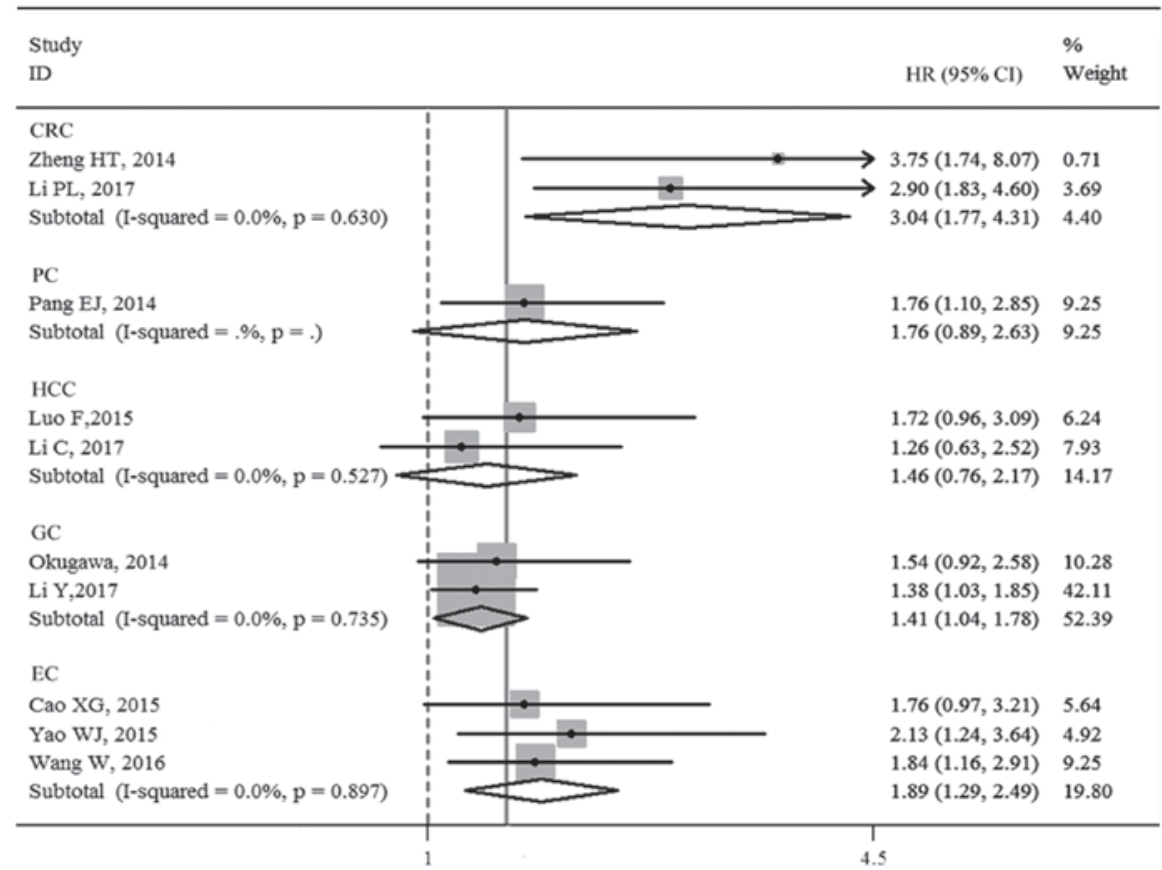

Figure 6. Forest plots for the analysis of the association between MALAT1 expression level and overall survival in different cancer types. Each square represents the HR point estimate and its size is proportional to the weight of the study. The dotted vertical line demonstrates the null value (hazard ratio=1), which represents no increased risk for the outcome. HR, hazard ratio; CI, confidence interval; CRC, colorectal cancer; PC, pancreatic cancer; HCC, hepatocellular carcinoma; GC, gastric cancer; EC, esophageal cancer. 

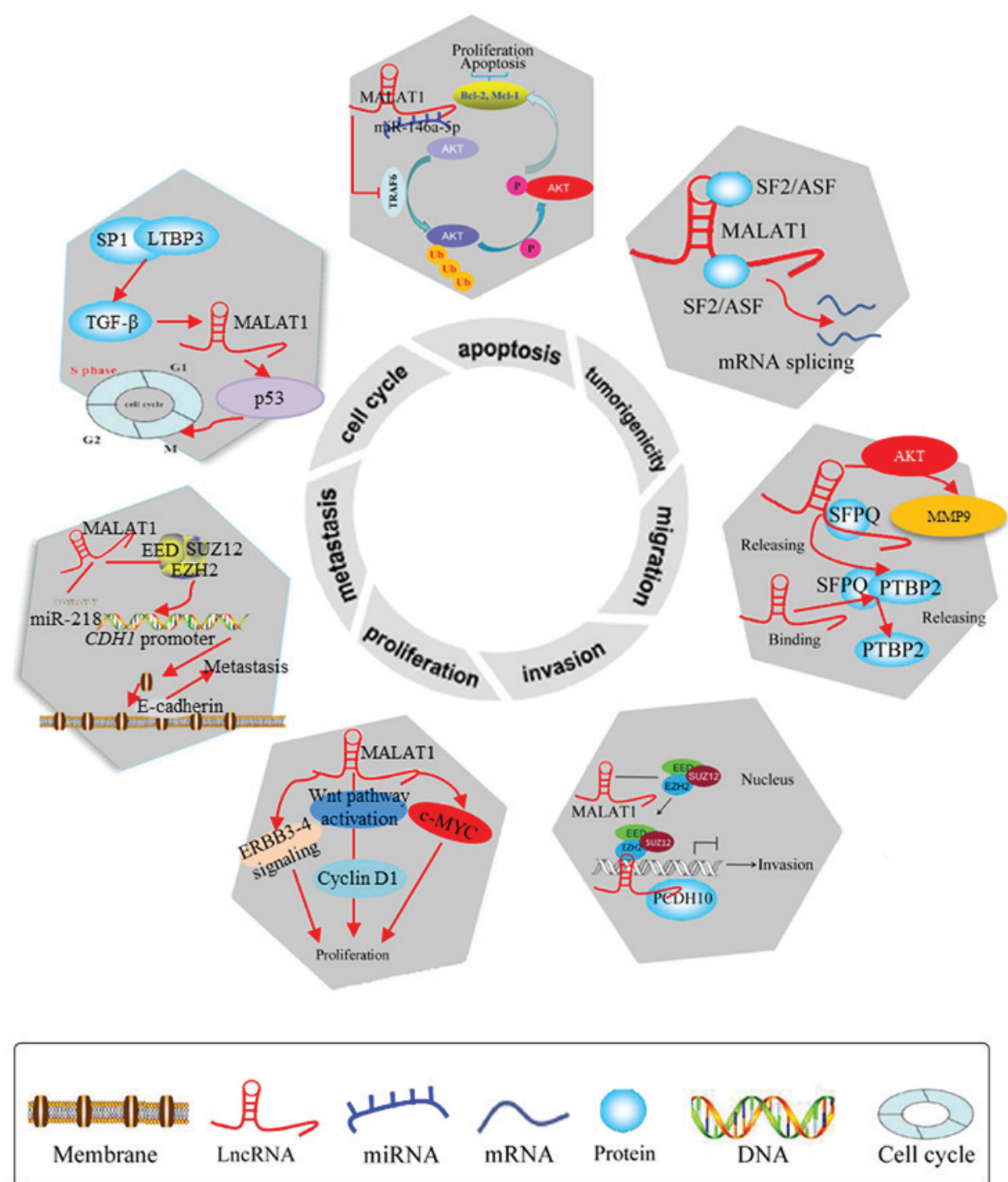

Figure 7. Association of MALAT1 expression levels and the development of digestive system malignancies via the regulation of cell apoptosis, cell cycle, cell invasion, migration, metastasis and tumorigenicity. TRAF6, tumor necrosis factor receptor associated factor 6; SF2, splicing factor 2; ASF, alternative splicing factor; SFPQ, splicing factor proline/glutamine rich; PTBP2, polypyrimidine tract binding protein 2; EED, embryonic ectoderm development; EZH2, enhancer of zeste 2 polycomb repressive complex 2 subunit; SUZ12, SUZ12 polycomb repressive complex 2 subunit; PCDH10, protocadherin 10; ERBB2, erb-b2 receptor tyrosine kinase 2; SP1, SP1 nuclear transcription factor; LTBP3, latent transforming growth factor $\beta$-binding protein 3 ; lncRNA, long non-coding RNA; TGF- $\beta$, transforming growth factor- $\beta$; MMP9, matrix metallopeptidase 9.

which indirectly influences mRNA splicing (41). In cancer migration studies, MALAT1 has been identified as one of the several important splicing factor proline/glutamine-rich (SFPQ)-binding RNAs. MALAT1 may competitively bind to SFPQ to release SFPQ from SFPQ/polypyrimidine tract binding protein 2 (PTBP2), which affects the AKT/matrix metallopeptidase 9 signaling pathway. This is critical for the regulation of cell migration (42). In cancer invasion studies, it has been revealed that enhancer of zeste 2 polycomb repressive complex 2 subunit (EZH2) is tethered by MALAT1 to the protocadherin 10 (PCDH10) locus, which promotes cancer metastasis and invasion by suppressing PCDH10 (25). Additionally, MALAT1 tethers EZH2 to the cadherin 1 promoter and suppresses miR-218, which promotes CRC cell EMT, metastasis and chemoresistance (37). Overall, MALAT1 mediates cancer cell proliferation, migration, invasion and metastasis, and may therefore be a potential prognostic factor for digestive system malignancies.

\section{Discussion}

Previously, a number of studies have demonstrated that lncRNAs participate in tumorigenesis and cancer prognosis. Certain lncRNAs, including HOX transcript antisense intergenic RNA, colon cancer-associated transcript 2, PVT1 oncogene, maternally expressed gene 3 and MALAT1 have been demonstrated to serve a role in cancer progression and metastasis in digestive system malignancies $(43,44)$. With advancements in high-throughput sequencing, lncRNAs have become a key interest for RNA biology studies. MALAT1 is abnormally expressed in human malignancies $(16,19,37,42)$. However, to the best of our knowledge, the independent prognostic role of MALAT1 in digestive system malignancies remains unclear. Therefore, the current study performed a meta-analysis to evaluate the clinical implication and prognostic value of MALAT1 in five principal digestive system malignancies. According to present knowledge, this is a 
meaningful meta-analysis to explore the association between MALAT1 expression and OS in human digestive system cancer types.

A previous study has provided certain information regarding the prognostic value of MALAT1 (45), however the current study provides a more recent update and performs subgroup analysis. The present meta-analysis included 12 studies that focused on digestive system cancer types. The prognostic value of MALAT1 in digestive system cancer was assessed. The present study demonstrated that high expression levels of MALAT1 were associated with poor OS in patients (HR, 1.62; 95\% CI, 1.35-1.88). Furthermore, subgroup analysis revealed a significant association between high expression of MALAT1 and disease prognosis, while country, type of cancer, sample size and analysis method did not significantly affect these pooled results. The heterogeneity $\mathrm{Q}$ statistic $(\mathrm{P}>0.05)$ and $\mathrm{I}^{2}$ value was $0.00 \%$, indicating no statistical evidence for heterogeneity among the selected studies. Both Begg's test and Egger's test revealed no publication bias, and sensitivity analysis supported the statistical power of the current study to investigate the independent prognostic role of MALAT1 for the OS of digestive system cancer. Particularly, elevated MALAT1 levels were significantly associated with decreased OS in the CRC subgroup (HR, 3.04; 95\% CI, 1.77-4.31). A number of studies have demonstrated that MALAT1 serves an important role in CRC (37,46-49).

MALAT1, regarded as one of the most common oncogenic lncRNAs, has also been identified to be highly expressed in other various cancer types, including hepatocellular carcinoma (50), non-small cell lung cancer (42), GC (36), pancreatic cancer (17,35), CRC (16), clear cell renal cell carcinoma (51), esophageal cancer $(52,53)$, gallbladder cancer (54), bladder cancer (55), osteosarcoma (56) and breast cancer $(57,58)$. A larger number of these studies were predominantly performed in Asian populations, with few taking place in European countries; the most common countries were China, Japan and Germany. These studies identified that MALAT1 regulates tumor cell viability, invasiveness, migration and EMT $(59,60)$. In addition, a number of studies have revealed that overexpression of MALAT1 is significantly associated with high-risk grade, metastasis and patients' survival in numerous cancer types in Asian populations $(37,39,61)$. Notably, a Chinese study identified a positive association between single nucleotide polymorphisms in MALAT1 and cancer risk, therefore, genetic variants in MALAT1 may serve crucial roles in the development of cancer in Chinese populations (62). The small variation in regions in which previous studies have been performed may be a limitation that decreases the applicability of the current results across different ethnicities.

LncRNAs regulate gene expression by a number of mechanisms, including transcription, posttranscriptional processing, chromatin modification, and protein function regulation (9). To provide further insights, the mechanism of action for MALAT1 in digestive system malignancies was summarized based on a systematic review. This revealed that MALAT1 is involved in the regulation of cancer cell apoptosis and proliferation, in addition to the induction of cell cycle arrest, tumorigenicity, migration, invasion and metastasis. This can be summarized as two modes of action for MALAT1. On one hand, an increasing number of studies have indicated that MALAT1 promotes cancer progression by regulating the expression of genes, including apoptosis-associated genes (Akt, Bax, Bcl2, and Mcl1) (34), cell cycle-associated genes (P53 and Mybl2) (40), EMT and metastasis-associated genes (ZEB1, ZEB2, Slug and E-cadherin) (25), cancer invasion-associated genes (EZH2 and PCDH10). On the other hand, MALAT1 has been identified to serve a role in alternative mRNA splicing. For example, MALAT1 promotes tumorigenicity by recruiting SF2/ASF, which subsequently indirectly influences mRNA splicing (41). In addition, MALAT1 affects cancer migration-associated splicing factors, including SFPQ and PTBP2 (42). In summary, there is an urgent requirement to determine the exact molecular mechanisms of MALAT1 in the progression of digestive system cancer types.

A number of limitations are unavoidable when performing a meta-analysis. Firstly, potential bias may exist due to the more frequent publication of positive results. Secondly, studies with small sample sizes may not adequately investigate potential associations. Thirdly, adjuvant therapeutic strategies are different in different types of digestive system malignancy, which may affect the postoperative survival of patients. In addition, potential bias may exist in the selection of studies, partly due to the language in which they are published. Finally, all studies included in the present meta-analysis were conducted in Asian populations, particularly, Chinese and Japanese populations. Furthermore, the current study attempted to conduct a multivariable analysis, which requests original data of the selected studies and helps determine the prognostic significance of MALAT1. However, due to various difficulties, multivariable analysis was not conducted. Therefore, the present conclusions regarding the prognostic significance of MALAT1 in different types of digestive system malignancy should be interpreted with caution.

In summary, the present meta-analysis demonstrated that the overexpression of MALAT1 is associated with poor prognosis in various types of digestive system malignancy, particularly CRC. This aids understanding on the potential prognostic significance of MALAT1 in clinical practice.

\section{Acknowledgements}

Not applicable.

\section{Funding}

The study was supported by the National Natural Science Foundation of China (grant no. 81372956), the Natural Science Foundation of Jiangsu Universities (grant no. KYLX16_1126) and the Doctoral Scientific Research Foundation of Jiangsu Province (grant no. 14B14).

\section{Availability of data and materials}

The datasets analyzed for the present study are available from the corresponding author upon reasonable request.

\section{Authors' contributions}

$\mathrm{CW}$ and JY conceived and designed the study. CW, QZ. and YH. searched and selected publications. CW, QZ, YH and 
JZ analyzed the data. CW and QZ prepared the figures. CW contributed materials/analysis tools. CW, and JY wrote and revised the paper. All authors critically reviewed the manuscript and approved the final draft.

\section{Ethics approval and consent to participate}

Not applicable. All the data/samples were collected from the articles in online databases and used anonymously.

\section{Patient consent for publication}

Not applicable.

\section{Competing interests}

The authors declare that they have no competing interests.

\section{References}

1. Torre LA, Bray F, Siegel RL, Ferlay J, Lortet-Tieulent J and Jemal A: Global cancer statistics, 2012. CA Cancer J Clin 65 : 87-108, 2015.

2. Nakagawa K, Tanaka K, Homma Y, Nojiri K, Kumamoto T, Takeda $\mathrm{K}$ and Endo I: Low infiltration of peritumoral regulatory $\mathrm{T}$ cells predicts worse outcome following resection of colorectal liver metastases. Ann Surg Oncol 22: 180-186, 2015.

3. Hou J, Zhou Y, Zheng Y, Fan J, Zhou W, Ng IO, Sun H, Qin L, Qiu S, Lee JM, et al: Hepatic RIG-I predicts survival and interferon-alpha therapeutic response in hepatocellular carcinoma. Cancer Cell 25: 49-63, 2014.

4. Ohlsson L, Israelsson A, Öberg Å, Palmqvist R, Stenlund H, Hammarström ML, Hammarström S and Lindmark G: Lymph node CEA and MUC2 mRNA as useful predictors of outcome in colorectal cancer. Int J Cancer 130: 1833-1843, 2012.

5. Raziee HR, Cardoso R, Seevaratnam R, Mahar A, Helyer L, Law $\mathrm{C}$ and Coburn N: Systematic review of the predictors of positive margins in gastric cancer surgery and the effect on survival. Gastric Cancer 15 (Suppl 1): S116-S124, 2012.

6. Xu T, Lin CM, Cheng SQ, Min J, Li L, Meng XM, Huang C, Zhang L, Deng ZY and Li J: Pathological bases and clinica impact of long noncoding RNAs in prostate cancer: A new budding star. Mol Cancer 17: 103, 2018.

7. Djebali S, Davis CA, Merkel A, Dobin A, Lassmann T, Mortazavi A, Tanzer A, Lagarde J, Lin W, Schlesinger F, et al: Landscape of transcription in human cells. Nature 489: 101-108, 2012.

8. Ma L, Bajic VB and Zhang Z: On the classification of long non-coding RNAs. RNA Biol 10: 925-933, 2013.

9. Gupta RA, Shah N, Wang KC, Kim J, Horlings HM, Wong DJ, Tsai MC, Hung T, Argani P, Rinn JL, et al: Long non-coding RNA HOTAIR reprograms chromatin state to promote cancer metastasis. Nature 464: 1071-1076, 2010.

10. Zhang X, Gejman R, Mahta A, Zhong Y, Rice KA, Zhou Y, Cheunsuchon P, Louis DN and Klibanski A: Maternally expressed gene 3, an imprinted noncoding RNA gene, is associated with meningioma pathogenesis and progression. Cancer Res 70: 2350-2358, 2010.

11. Fu M, Zou C, Pan L, Liang W, Qian H, Xu W, Jiang P and Zhang X: Long noncoding RNAs in digestive system cancers: Functional roles, molecular mechanisms, and clinical implications (Review). Oncol Rep 36: 1207-1218, 2016.

12. Wang KC and Chang HY: Molecular mechanisms of long noncoding RNAs. Mol Cell 43: 904-914, 2011.

13. Thomassen I, van Gestel YR, van Ramshorst B, Luyer MD, Bosscha K, Nienhuijs SW, Lemmens VE and de Hingh IH: Peritoneal carcinomatosis of gastric origin: A population-based study on incidence, survival and risk factors. Int J Cancer 134 622-628, 2014

14. Shen L, Chen L, Wang Y, Jiang X, Xia H and Zhuang Z: Long noncoding RNA MALAT1 promotes brain metastasis by inducing epithelial-mesenchymal transition in lung cancer. J Neurooncol 121: 101-108, 2015.
15. Lai MC, Yang Z, Zhou L, Zhu QQ, Xie HY, Zhang F, Wu LM, Chen LM and Zheng SS: Long non-coding RNA MALAT-1 overexpression predicts tumor recurrence of hepatocellular carcinoma after liver transplantation. Med Oncol 29: 1810-1816, 2012.

16. Zheng HT, Shi DB, Wang YW, Li XX, Xu Y, Tripathi P, Gu WL, Cai GX and Cai SJ: High expression of lncRNA MALAT1 suggests a biomarker of poor prognosis in colorectal cancer. Int J Clin Exp Pathol 7: 3174-3181, 2014.

17. Liu JH, Chen G, Dang YW, Li CJ and Luo DZ: Expression and prognostic significance of lncRNA MALAT1 in pancreatic cancer tissues. Asian Pac J Cancer Prev 15: 2971-2977, 2014.

18. Li Y, Wu Z, Yuan J, Sun L, Lin L, Huang N, Bin J, Liao Y and Liao W: Long non-coding RNA MALAT1 promotes gastric cancer tumorigenicity and metastasis by regulating vasculogenic mimicry and angiogenesis. Cancer Lett 395: 31-44, 2017.

19. Cao X, Zhao R, Chen Q, Zhao Y, Zhang B, Zhang Y, Yu J, Han G, Cao W, Li J and Chen X: MALAT1 might be a predictive marker of poor prognosis in patients who underwent radical resection of middle thoracic esophageal squamous cell carcinoma. Cancer Biomark 15: 717-723, 2015

20. Wang JZ, Xiang JJ, Wu LG, Bai YS, Chen ZW, Yin XQ, Wang Q, Guo WH, Peng Y, Guo $\mathrm{H}$ and $\mathrm{Xu} \mathrm{P}$ : A genetic variant in long non-coding RNA MALAT1 associated with survival outcome among patients with advanced lung adenocarcinoma: A survival cohort analysis. BMC Cancer 17: 167, 2017.

21. Jadaliha M, Zong X, Malakar P, Ray T, Singh DK, Freier SM, Jensen T, Prasanth SG, Karni R, Ray PS and Prasanth KV: Functional and prognostic significance of long non-coding RNA MALAT1 as a metastasis driver in ER negative lymph node negative breast cancer. Oncotarget 7: 40418-40436, 2016.

22. Wang Y, Zhang Y, Yang T, Zhao W, Wang N, Li P, Zeng X and Zhang W: Long non-coding RNA MALAT1 for promoting metastasis and proliferation by acting as a ceRNA of miR-144-3p in osteosarcoma cells. Oncotarget 8: 59417-59434, 2017.

23. Liu M, Sun W, Liu Y and Dong X: The role of IncRNA MALAT1 in bone metastasis in patients with non-small cell lung cancer. Oncol Rep 36: 1679-1685, 2016.

24. Zhang TH, Liang LZ, Liu XL, Wu JN, Su K, Chen JY, Zheng QY, Huang HZ and Liao GQ: Long non-coding RNA MALAT1 interacts with miR-124 and modulates tongue cancer growth by targeting JAG1. Oncol Rep 37: 2087-2094, 2017.

25. Qi Y, Ooi HS, Wu J, Chen J, Zhang X, Tan S, Yu Q, Li YY, Kang Y, Li H, et al: MALAT1 long ncRNA promotes gastric cancer metastasis by suppressing PCDH10. Oncotarget 7: 12693-12703, 2016.

26. Ji Q, Liu X, Fu X, Zhang L, Sui H, Zhou L, Sun J, Cai J, Qin J, Ren J and Li Q: Resveratrol inhibits invasion and metastasis of colorectal cancer cells via MALAT1 mediated Wnt/ $\beta$-catenin signal pathway. PLoS One 8: e78700, 2013.

27. Deng J, Zhang R, Pan Y, Wang B, Wu L, Jiao X, Bao T, Hao X and Liang $\mathrm{H}$ : Comparison of the staging of regional lymph nodes using the sixth and seventh editions of the tumor-node-metastasis (TNM) classification system for the evaluation of overall survival in gastric cancer patients: Findings of a case-control analysis involving a single institution in China. Surgery 156: 64-74, 2014.

28. Tierney JF, Stewart LA, Ghersi D, Burdett S and Sydes MR: Practical methods for incorporating summary time-to-event data into meta-analysis. Trials 8: 16, 2007.

29. Williamson PR, Smith CT, Hutton JL and Marson AG: Aggregate data meta-analysis with time-to-event outcomes. Stat Med 21: 3337-3351, 2002

30. Wang C, Yang L, Wang S, Zhang Z, Yu Y, Wang M, Cromie M, Gao W and Wang SL: The classic EDCs, phthalate esters and organochlorines, in relation to abnormal sperm quality: A systematic review with meta-analysis. Sci Rep 6: 19982, 2016.

31. Peters JL, Sutton AJ, Jones DR, Abrams KR and Rushton L: Contour-enhanced meta-analysis funnel plots help distinguish publication bias from other causes of asymmetry. J Clin Epidemiol 61: 991-996, 2008.

32. Begg CB and Mazumdar M: Operating characteristics of a rank correlation test for publication bias. Biometrics 50: 1088-1101, 1994.

33. Luo F, Sun B, Li H, Xu Y, Liu Y, Liu X, Lu L, Li J, Wang Q, Wei S, et al: A MALAT1/HIF-2 $\alpha$ feedback loop contributes to arsenite carcinogenesis. Oncotarget 7: 5769-5787, 2016.

34. Li C, Miao R, Liu S, Wan Y, Zhang S, Deng Y, Bi J, Qu K, Zhang J and Liu C: Down-regulation of miR-146b-5p by long noncoding RNA MALAT1 in hepatocellular carcinoma promotes cancer growth and metastasis. Oncotarget 8: 28683-28695, 2017. 
35. Pang EJ, Yang R, Fu XB and Liu YF: Overexpression of long non-coding RNA MALAT1 is correlated with clinical progression and unfavorable prognosis in pancreatic cancer. Tumour Biol 36: 2403-2407, 2015.

36. Okugawa Y, Toiyama Y, Hur K, Toden S, Saigusa S, Tanaka K, Inoue $\mathrm{Y}$, Mohri Y, Kusunoki M, Boland CR and Goel A: Metastasis-associated long non-coding RNA drives gastric cancer development and promotes peritoneal metastasis. Carcinogenesis 35: 2731-2739, 2014.

37. Li P, Zhang X, Wang H, Wang L, Liu T, Du L, Yang Y and Wang C: MALAT1 is associated with poor response to oxaliplatin-based chemotherapy in colorectal cancer patients and promotes chemoresistance through EZH2. Mol Cancer Ther 16 739-751, 2017.

38. Yao W, Bai Y, Li Y, Guo L, Zeng P, Wang Y, Qi B, Liu S, Qin X, Li Y and Zhao B: Upregulation of MALAT-1 and its association with survival rate and the effect on cell cycle and migration in patients with esophageal squamous cell carcinoma. Tumour Biol 37: 4305-4312, 2016.

39. Wang W, Zhu Y, Li S, Chen X, Jiang G, Shen Z, Qiao Y, Wang L, Zheng $\mathrm{P}$ and Zhang Y: Long noncoding RNA MALAT1 promotes malignant development of esophageal squamous cell carcinoma by targeting $\beta$-catenin via Ezh2. Oncotarget 7: 25668-25682, 2016.

40. Tripathi V, Shen Z, Chakraborty A, Giri S, Freier SM, Wu X, Zhang Y, Gorospe M, Prasanth SG, Lal A and Prasanth KV: Long noncoding RNA MALAT1 controls cell cycle progression by regulating the expression of oncogenic transcription factor B-MYB. PLoS Genet 9: e1003368, 2013.

41. Wang J, Su L, Chen X, Li P, Cai Q, Yu B, Liu B, Wu W and Zhu Z: MALAT1 promotes cell proliferation in gastric cancer by recruiting SF2/ASF. Biomed Pharmacother 68: 557-564, 2014.

42. Ji Q, Zhang L, Liu X, Zhou L, Wang W, Han Z, Sui H, Tang Y, Wang Y, Liu N, et al: Long non-coding RNA MALAT1 promotes tumour growth and metastasis in colorectal cancer through binding to SFPQ and releasing oncogene PTBP2 from SFPQ/PTBP2 complex. Br J Cancer 111: 736-748, 2014.

43. Li CH and Chen Y: Targeting long non-coding RNAs in cancers: Progress and prospects. Int J Biochem Cell Biol 45: 1895-1910, 2013.

44. Shi X, Sun M, Liu H, Yao Y and Song Y: Long non-coding RNAs: A new frontier in the study of human diseases. Cancer Lett 339: 159-166, 2013

45. Zhai H, Li XM, Maimaiti A, Chen QJ, Liao W, Lai HM, Liu F and Yang YN: Prognostic value of long noncoding RNA MALAT1 in digestive system malignancies. Int J Clin Exp Med 8: 18099-18106, 2015.

46. Xiong Y, Wang J, Zhu H, Liu L and Jiang Y: Chronic oxymatrine treatment induces resistance and epithelialmesenchymal transition through targeting the long non-coding RNA MALAT1 in colorectal cancer cells. Oncol Rep 39: 967-976, 2018.

47. Li Y, Bao C, Gu S, Ye D, Jing F, Fan C, Jin M and Chen K: Associations between novel genetic variants in the promoter region of MALAT1 and risk of colorectal cancer. Oncotarget 8: 92604-92614, 2017.

48. Li Q, Dai Y, Wang F and Hou S: Differentially expressed long non-coding RNAs and the prognostic potential in colorectal cancer. Neoplasma 63: 977-983, 2016.

49. Hu ZY, Wang XY, Guo WB, Xie LY, Huang YQ, Liu YP, Xiao LW, Li SN, Zhu HF, Li ZG and Kan H: Long non-coding RNA MALAT1 increases AKAP-9 expression by promoting SRPK1-catalyzed SRSF1 phosphorylation in colorectal cancer cells. Oncotarget 7: 11733-11743, 2016.
50. Liu D, Zhu Y, Pang J, Weng X, Feng X and Guo Y: Knockdown of long non-coding RNA MALAT1 inhibits growth and motility of human hepatoma cells via modulation of miR-195. J Cell Biochem 119: 1368-1380, 2018.

51. Kidane B, Chadi SA, Kanters S, Colquhoun PH and Ott MC: Local resection compared with radical resection in the treatment of T1N0M0 rectal adenocarcinoma: A systematic review and meta-analysis. Dis Colon Rectum 58: 122-140, 2015.

52. Hu L, Wu Y, Tan D, Meng H, Wang K, Bai Y and Yang K: Up-regulation of long noncoding RNA MALAT1 contributes to proliferation and metastasis in esophageal squamous cell carcinoma. J Exp Clin Cancer Res 34: 7, 2015.

53. Huang C, Yu Z, Yang H and Lin Y: Increased MALAT1 expression predicts poor prognosis in esophageal cancer patients. Biomed Pharmacother 83: 8-13, 2016.

54. Wang SH, Zhang WJ, Wu XC, Weng MZ, Zhang MD, Cai Q, Zhou D, Wang JD and Quan ZW: The lncRNA MALAT1 functions as a competing endogenous RNA to regulate MCL-1 expression by sponging miR-363-3p in gallbladder cancer. J Cell Mol Med 20: 2299-2308, 2016

55. Fan Y, Shen B, Tan M, Mu X, Qin Y, Zhang F and Liu Y: TGF- $\beta$-induced upregulation of malat1 promotes bladder cancer metastasis by associating with suz12. Clin Cancer Res 20: 1531-1541, 2014.

56. Dong Y, Liang G, Yuan B, Yang C, Gao R and Zhou X: MALAT1 promotes the proliferation and metastasis of osteosarcoma cells by activating the PI3K/Akt pathway. Tumour Biol 36: 1477-1486, 2015.

57. Huang NS, Chi YY, Xue JY, Liu MY, Huang S, Mo M, Zhou SL and $\mathrm{Wu} \mathrm{J}$ : Long non-coding RNA metastasis associated in lung adenocarcinoma transcript 1 (MALAT1) interacts with estrogen receptor and predicted poor survival in breast cancer. Oncotarget 7: 37957-37965, 2016.

58. Miao Y, Fan R, Chen L and Qian H: Clinical significance of long non-coding RNA MALAT1 expression in tissue and serum of breast cancer. Ann Clin Lab Sci 46: 418-424, 2016.

59. Yoshimoto R, Mayeda A, Yoshida M and Nakagawa S: MALAT1 long non-coding RNA in cancer. Biochim Biophys Acta 1859: 192-199, 2016.

60. Jiao F, Hu H, Yuan C, Wang L, Jiang W, Jin Z, Guo Z and Wang L: Elevated expression level of long noncoding RNA MALAT-1 facilitates cell growth, migration and invasion in pancreatic cancer. Oncol Rep 32: 2485-2492, 2014

61. Xia H, Chen Q, Chen Y, Ge X, Leng W, Tang Q, Ren M, Chen L, Yuan D, Zhang Y, et al: The IncRNA MALAT1 is a novel biomarker for gastric cancer metastasis. Oncotarget 7: 56209-56218, 2016.

62. Peng R, Luo C, Guo Q, Cao J, Yang Q, Dong K, Wang S, Wang K and Song C: Association analyses of genetic variants in long non-coding RNA MALAT1 with breast cancer susceptibility and mRNA expression of MALAT1 in Chinese Han population. Gene 642: 241-248, 2018.

This work is licensed under a Creative Commons Attribution-NonCommercial-NoDerivatives 4.0 International (CC BY-NC-ND 4.0) License. 